\title{
HYPOXIC ISCHAEMIC ENCEPHALOPATHY: ASSESSMENT OF SEVERITY BY MR IMAGING
}

\author{
Sajitha Arimbrathodi1, Vadakooth Raman Rajendran², Jineesh Thottath ${ }^{3}$, Juvaina Puthiyakam ${ }^{4}$, Saanida Monthampally5
}

1 Junior Resident, Department of Radiodiagnosis, Government Medical College, Kozhikode. 2 Professor \& HOD, Department of Radiodiagnosis, Government Medical College, Kozhikode. ${ }^{3}$ Assistant Professor, Department of Radiodiagnosis, Government Medical College, Kozhikode. ${ }^{4}$ Assistant Professor, Department of Radiodiagnosis, Government Medical College, Kozhikode. ${ }_{5}^{5}$ Assistant Professor, Department of Radiodiagnosis, Government Medical College, Kozhikode.

\section{ABSTRACT}

\section{CONTEXT}

Perinatal asphyxia happens in 2 to 10 per 1000 newborns that are born at term, and more for those that are born prematurely and making one of the leading cause of death worldwide.

\section{AIM}

To study the pattern of involvement of brain in HIE influenced by nature of insult and to assess the severity of brain injury by MR imaging and correlate with clinical staging (Sarnat criteria).

\section{METHODOLOGY}

43 cases with history of perinatal asphyxia were included in this study by applying the inclusion criteria and exclusion criteria. Signal changes in T1 and T2 weighted images and diffusion weighted were assessed and recorded.

\section{RESULTS AND INTERPRETATIONS}

After MR imaging, most of the Sarnat Stage I and II patients were mild and stage III patients were severe. Of the all clinically diagnosed having HIE, $25.6 \%$ cases were normal. Patients with clinically mild hypotension $>1 / 2(54.2 \%)$ of them were found to be normal after imaging. Patients with clinically moderate hypotension, $20 \%$ were normal at MRI; and $>1 / 2$ (52\%) of them showed only mild changes and $12 \%$ showed severe involvement. Term babies with mild encephalopathy shows more involvement of periventricular white matter than subcortical white matter. Clinically severe encephalopathy correlates with abnormal basal ganglia - thalamic lesions in term babies and germinal matrix haemorrhage in preterm babies. Clinically moderate encephalopathy correlates with signal changes in posterior limb of internal capsule and perirolandic white matter. Prematurity increases the susceptibility of brain to changes caused by hypoxia. No significant association noted between sex and birth weight with abnormal MR imaging findings. The low Apgar score shows significant association with severity of brain involvement. Seizure and its onset also appears to be a deciding parameter in severity of brain injury.

\section{KEYWORDS}

HIE - Hypoxic Ischaemic Encephalopathy, MRI - Magnetic Resonance Imaging, DWI - Diffusion Weighted Imaging, T1WI - T1 Weighted Imaging, T2WI - T2 Weighted Imaging, ADC - Apparent Diffusion Coefficient.

HOW TO CITE THIS ARTICLE: Arimbrathodi S, Rajendran VR, Thottath J, et al. Hypoxic ischaemic encephalopathy: assessment of severity by MR imaging. J. Evolution Med. Dent. Sci. 2016;5(63):4468-4475, DOI: 10.14260/jemds/2016/1019

\section{INTRODUCTION}

Perinatal asphyxia happens in 2 to 10 per 1000 newborns that are born at term and more for those that are born prematurely. A 2008 bulletin from the World Health Organization estimates that a total of 900,001 total infants die each year from birth asphyxia, making it a leading cause of death for newborns (As per 2013 statistics).

Magnetic Resonance Imaging (MRI), an increasingly available and non-invasive examination (may be performed during the child's physiological sleep), which unlike the computed tomography does not involve the child's exposure to X-ray. MRI allows to reveal a number of lesions associated with HIE that are invisible on the ultrasound scans.

Financial or Other, Competing Interest: None.

Submission 19-05-2016, Peer Review 22-07-2016,

Acceptance 28-07-2016, Published 08-08-2016.

Corresponding Author:

Dr. Sajitha Arimbrathodi,

Kanhirakkandi (H) Puthur Pallikkal P.O,

Thenhipalam, (via),

Malappuram-673636, Kerala, India.

E-mail: drsajithayasir@gmail.com

DOI: $10.14260 /$ jemds/2016/1019
Another MRI advantage is the possibility to predict the clinical consequences of hypoxic-ischaemic lesions. Magnetic Resonance Imaging (MRI) with its ability to detect certain abnormalities of tissue water in response to injury has been one of the widely used tool of diagnosis in both paediatric clinical and experimental application.

\section{AIMS AND OBJECTIVES}

- To study the pattern of involvement of brain in HIE influenced by nature of insult.

- $\quad$ To assess the severity of brain injury by MR imaging and correlate with clinical staging (Sarnat criteria).

\section{METHODS}

Study Design: Descriptive study.

Study Setting: Radiology Department Govt. Medical College, Kozhikode.

Study Period: Data collection was done in January 2013February 2014.

Sample Size: 43 subjects. 


\section{Inclusion Criteria}

Newborns with perinatal asphyxia and signs suggestive of HIE, who are undergoing imaging in our institution.

\section{Exclusion Criteria}

Newborns with congenital malformations and congenital infections.

\section{Study Method}

- $\quad$ MRI with a 1.5 T MR system (GE SIGNA HDXT).

- $\quad$ Axial (FSE) pulse sequence T1WI - (TR/TE, 205-730/4-16 $\mathrm{ms}$ ) and T2WI - (TR/TE, 1553-5897/80-200 ms) fast spin echo, fluid attenuated inversion recovery sequences (TR/TE/TI, 8000/110-120/1860-2000 ms), T2WI in sagittal plane and Gradient Echo Planar Image (EPI) DWI pulse, b-value 0,600 second/mm2.

- $\quad \mathrm{ADC}$ values are calculated within the lesion, diameter of ROI - $31.2 \mathrm{~mm}$.

\section{Statistical Analysis}

- $\quad$ SPSS statistical software.

- $\quad$ P value $</=0.05$ ? ?statistically significant difference.

\section{STUDY METHODOLOGY}

After getting informed consent from the parent, the sociodemographic details and the antenatal details of the mother, the clinical symptoms of asphyxiated newborn, history of seizure, feeding difficulty, reflex, tone, birth weight, Apgar, NND of imaging and maturity at birth are collected. They were included if they fulfilled the following criteria (Suggestive of perinatal asphyxia).

1. Signs of foetal distress before delivery (Abnormal cardiotocographic recording such as decreased variability, late deceleration, baseline bradycardia or Meconium stained liquor).

2. Apgar score of $<7$ after 5 minutes.

3. Clinical signs of hypoxic-ischaemic encephalopathy.

\section{Clinical Criteria for Diagnosis of HIE}

- $\quad$ Evidence of foetal distress (Abnormal CTG, Meconium stained liquor).

- Low Apgar at birth.

- Necessity of resuscitation.

- Neurological signs.

\section{Sarnat Clinical Grading of HIE}

Mild-irritability

- Alternating levels of consciousness.

- Poor feeding.

- Exaggerated moro/tendon reflexes.

- Muscle tone - normal to increase.

- Seizure - absent.

\section{Moderate}

- Lethargic.

- Exaggerated tendon reflexes.

- Muscle tone - decreased.

- Seizure within $24 \mathrm{hrs}$.

Severe

- Coma.

- Flaccid tone.

- Seizures.

- Brainstem and autonomic dysfunction.

\section{MRI Criteria}

After doing MRI, ischaemic injury has to be appreciated by detecting abnormal signal intensities in the following areas Subcortical white matter and (+/_) cortex, Posterior Limb of Internal Capsule (PLIC), Perirolandic White Matter (PRWM), Periventricular White Matter (PVWM), basal ganglia, thalami and brainstem. Data was entered as T1 hypo/hyper, T2 hypo/hyper, DWI+/_. Cases were classified as mild, moderate or severe. The pattern of injury was classified according to the abnormalities seen, as previously described by Christine P. Chao et al. ${ }^{1}$

In term babies SCW+/_C-taken as mild, PRWM and PLIC Moderate and brainstem, basal ganglia and thalami-severe. In preterm babies PVWM-mild germinal matrix bleed/basal ganglia and thalami lesions-severe.

\section{Ethics}

The study was approved by the Institutional Research Committee and Ethics Committee of Government Medical College, Kozhikode, Kerala, India.

\section{Statistics}

The Null Hypothesis (H0) assumes that there is no association between the predictor and outcome variables in the study population. When we conclude that there is statistical significance, the $\mathrm{P}$ value tells us what the probability is that our conclusion is wrong when in fact $\mathrm{HO}$ is correct. The lower the $\mathrm{P}$ value, the less likely that our rejection of $\mathrm{H} 0$ is erroneous. By convention, most analysts will not claim that they have found statistical significance if there is more than a $5 \%$ chance of being wrong $(\mathrm{P}=0.05)$.

\section{RESULTS}

\begin{tabular}{|c|c|c|}
\hline & Frequency & Percent \\
\hline Preterm & 6 & 14.0 \\
\hline Term & 37 & 86.0 \\
\hline Total & $\mathbf{4 3}$ & $\mathbf{1 0 0 . 0}$ \\
\hline \multicolumn{2}{|c|}{ Table 1: Frequency Distributions } \\
by Gestational Age \\
\hline
\end{tabular}

In this study group which comprised of total number of 43 newborn babies, 37 (86\%) cases were term and 6 (14\%) cases were preterm.

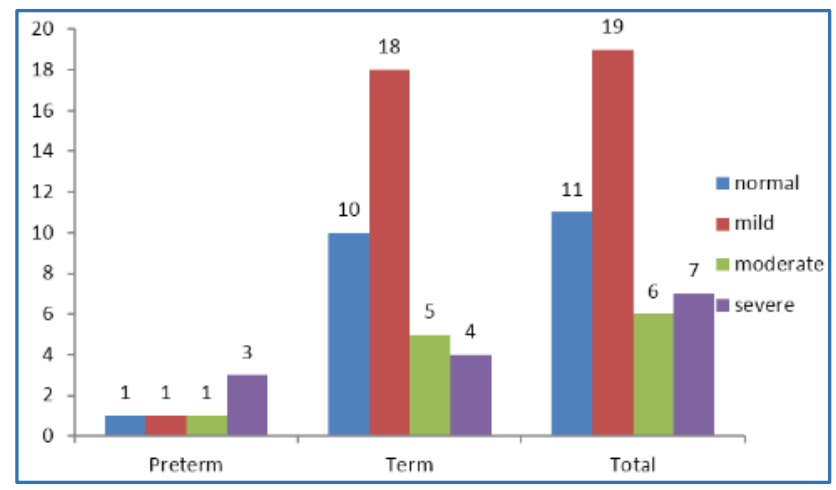

Fig. 1: Frequency Distribution of MRI Findings According to Gestational Age

Severity of brain injury in HIE is found to be increased in preterm newborns, ( $50 \%$ were severe), whereas majority of term babies showed only mild involvement (48\%). On 
performing $\mathrm{X}^{2}$ test to check an association, the $\mathrm{P}$ value was found to be 0.05 , which is statistically significant. So that according to this study, there is significant association between foetal maturity and brain injury in birth asphyxia and the chance of finding a severe MR abnormality is definitely high.

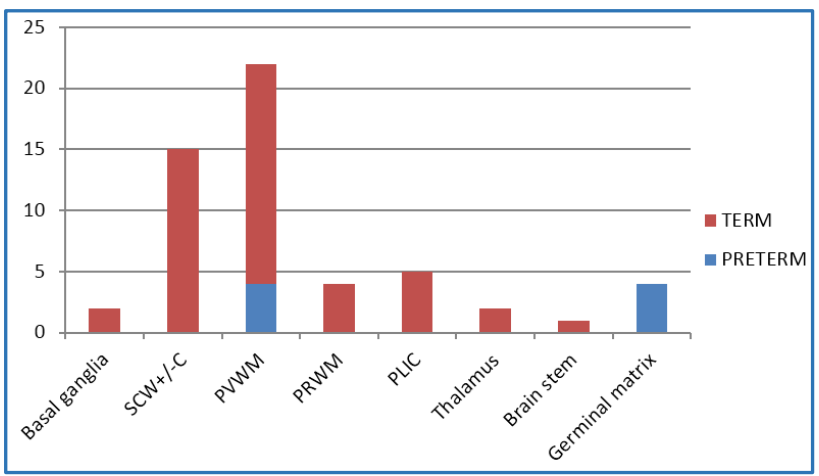

Fig. 2: Distribution of Areas of Brain Involvement in Preterm and Term

\begin{tabular}{|c|c|c|}
\hline Study Population & Number & Percentage \\
\hline Female & 16 & 37.2 \\
\hline Male & 27 & 62.8 \\
\hline Total & $\mathbf{4 3}$ & $\mathbf{1 0 0 . 0}$ \\
\hline \multicolumn{2}{|c|}{ Table 2: Sex Distribution } \\
\hline
\end{tabular}

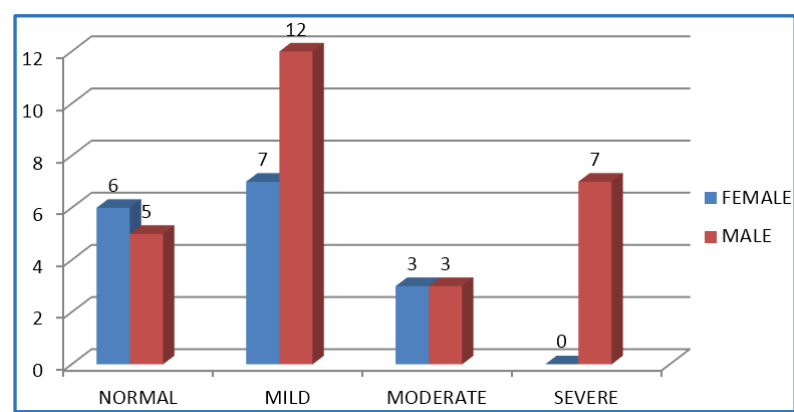

Fig. 3: Frequency Distribution of MRI Findings According to Sex

Severe involvement of HIE is common in males compared to females. However, $\mathrm{X}^{2}$ test to determine association between sex and MRI finding revealed $P$ value of $0.112(>0.05)$. Hence, according to this study, there is no statistically significant association between sex of the patient and chance of finding an abnormal MRI pattern in patients with HIE.

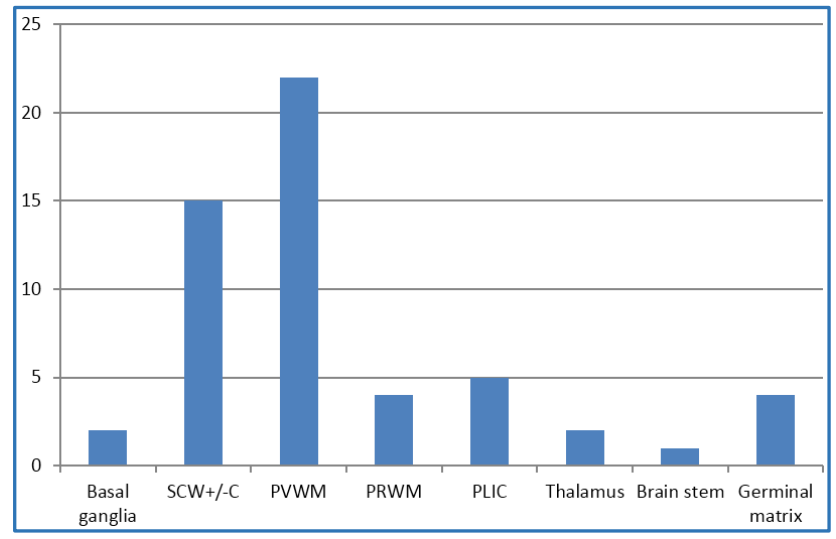

Fig. 4: Distribution of Areas of Brain Involved
It is observed that most commonly affected areas are periventricular white matter followed by cortex +/subcortical white matter.

\begin{tabular}{|c|c|c|}
\hline Birth Weight & Frequency & Percent \\
\hline $2.5 \mathrm{~kg}$ or Less & 16 & 37.2 \\
\hline$>2.5 \mathrm{~kg}$ & 27 & 62.8 \\
\hline Total & $\mathbf{4 3}$ & $\mathbf{1 0 0 . 0}$ \\
\hline \multicolumn{2}{|c|}{ Table 3: Birth Weight } \\
\hline
\end{tabular}

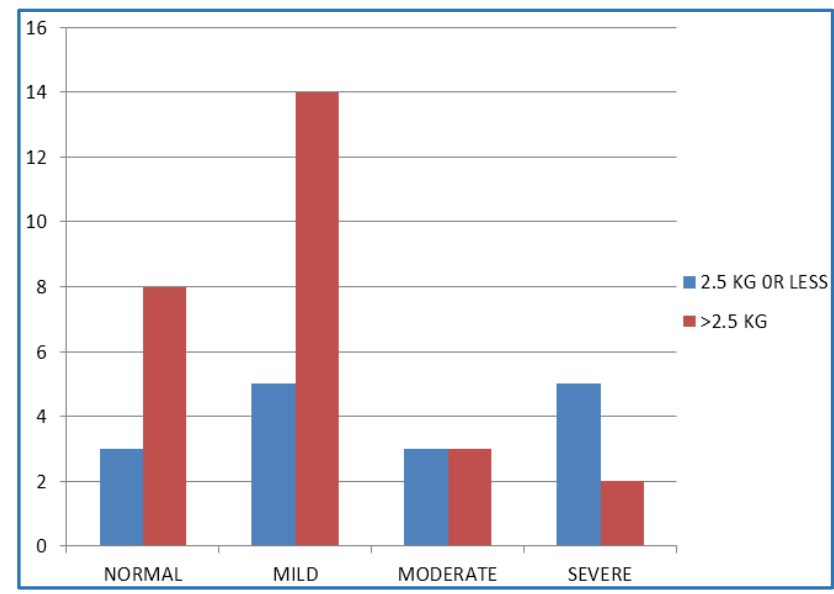

Fig. 5: Frequency Distribution of MRI Findings According to Birth Weight

Babies with low birth weight appear to be more commonly affected and show severe brain involvement on comparison to normal birth weight babies. $P$ value for this association was 0.115 ( $>0.05)$. According to this study, there is no statistically significant association between birth weight and abnormal MR finding in patients with HIE.

\begin{tabular}{|c|c|c|}
\hline Apgar Score & Frequency & Percent \\
\hline 7 or less than 7 & 15 & 34.9 \\
\hline More than 7 & 28 & 65.1 \\
\hline Total & $\mathbf{4 3}$ & $\mathbf{1 0 0 . 0}$ \\
\hline \multicolumn{2}{|c|}{ Table 4: Apgar Score } \\
\hline
\end{tabular}

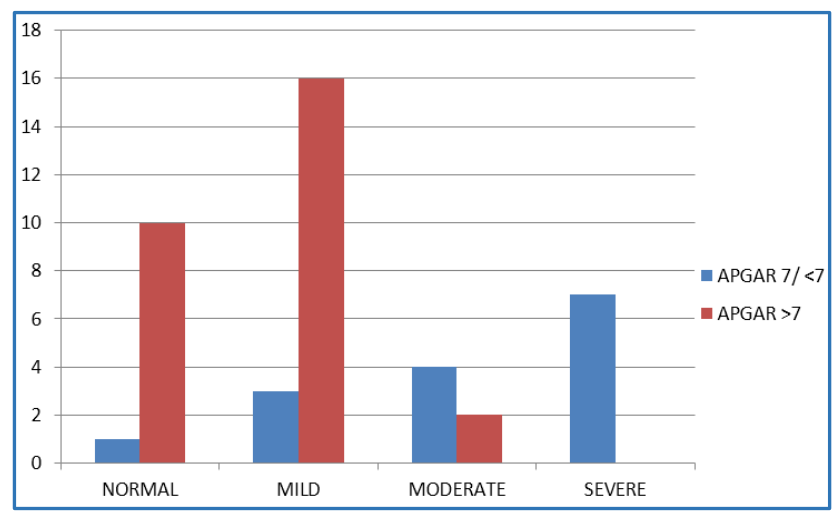

Fig. 6: Frequency Distribution of MRI Findings According to Apgar Score

Babies with low Apgar at 5 minutes appear to be more commonly affected and show severe MRI abnormalities. On performing $\mathrm{X}^{2}$ test to detect association between Apgar and MRI findings revealed $P$ value of $0.01(<0.05)$, which appears to be significant. So that according to this study, there is significant association between low Apgar score and brain injury in birth asphyxia and the chance of finding a severe MR abnormality is definitely high. 


\begin{tabular}{|c|c|c|c|c|c|c|c|c|}
\hline $\begin{array}{c}\text { Apgar } \\
\text { Score }\end{array}$ & $\begin{array}{c}\text { Basal } \\
\text { Ganglia }\end{array}$ & SCW+/C & PVWM & PRWM & PLIC & THALAMU & $\begin{array}{c}\text { Brain } \\
\text { Stem }\end{array}$ & $\begin{array}{c}\text { Germinal } \\
\text { Matrix }\end{array}$ \\
\hline 7 OR $<7$ & 2 & 5 & 7 & 3 & 3 & 2 & 1 & 4 \\
\hline$>7$ & - & 10 & 15 & 1 & 2 & - & - & - \\
\hline \multicolumn{8}{|c|}{ Table 5: Comparison of Areas Involved in MRI and Apgar Score } \\
\hline
\end{tabular}

\begin{tabular}{|c|c|c|}
\hline Seizure & Frequency & Percent \\
\hline No Seizure & 17 & 39.5 \\
\hline Within 24 Hours & 20 & 46.5 \\
\hline After 24 Hours & 6 & 14.0 \\
\hline Total & 43 & 100.0 \\
\hline \multicolumn{3}{|c|}{ Table 6: Frequency Distribution of Seizure } \\
\hline
\end{tabular}

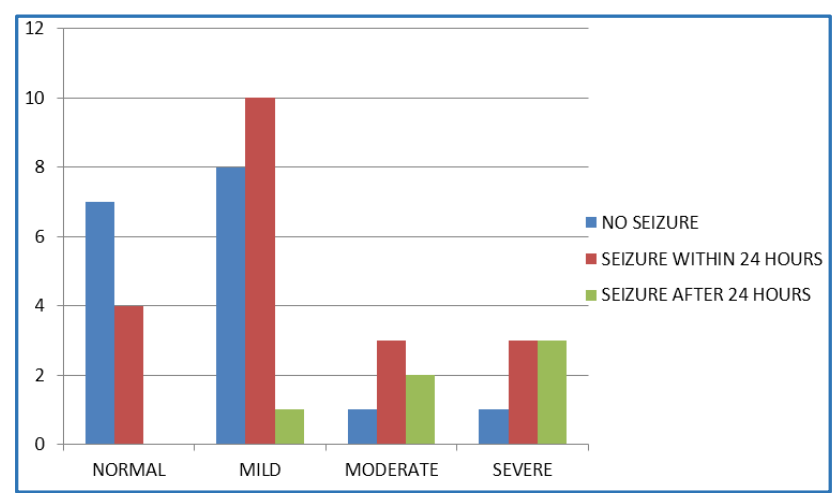

Fig. 7: Frequency Distribution of MRI Findings According to Seizure

Those having seizure within 24 hours, $80 \%$ were having abnormal MR findings. Out of this, majority were mild (62.2\%); 18.7\% were severe. Those having seizure after 24 hours, all cases showed abnormal MRI finding and about 50\% of them were severe. Out of all cases having seizure irrespective of time of onset, $84.8 \%$ showed involvement in MRI and delayed onset of seizure is correlating with severity of brain involvement. $\mathrm{P}$ value for this association was 0.05 , which appears to be significant.

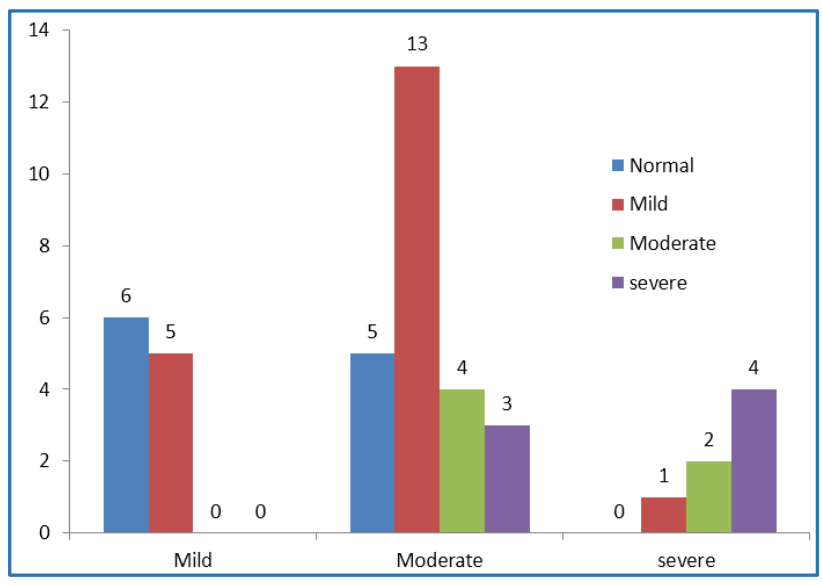

Fig. 8: Correlation between Sarnat's Grading and MR Diagnosis

When the $\mathrm{X}^{2}$ test was done to find out the association between clinical grading and MR diagnosis, the $\mathrm{P}$ value was found to be $0.004(<0.005)$, which appears to be significant.

To interpret that there is $>50 \%$ chance of getting normal MRI study even in patients clinically graded as mildly affected. And only $<50 \%$ shows mild brain injury in this group. In patients clinically having moderate HIE, there is a chance of finding a normal MRI study in $20 \%$ cases and mild involvement is $50 \%$. In those who are clinically severe, $>57 \%$ had severe involvement in MR and none were normal.

\section{Representative Cases}

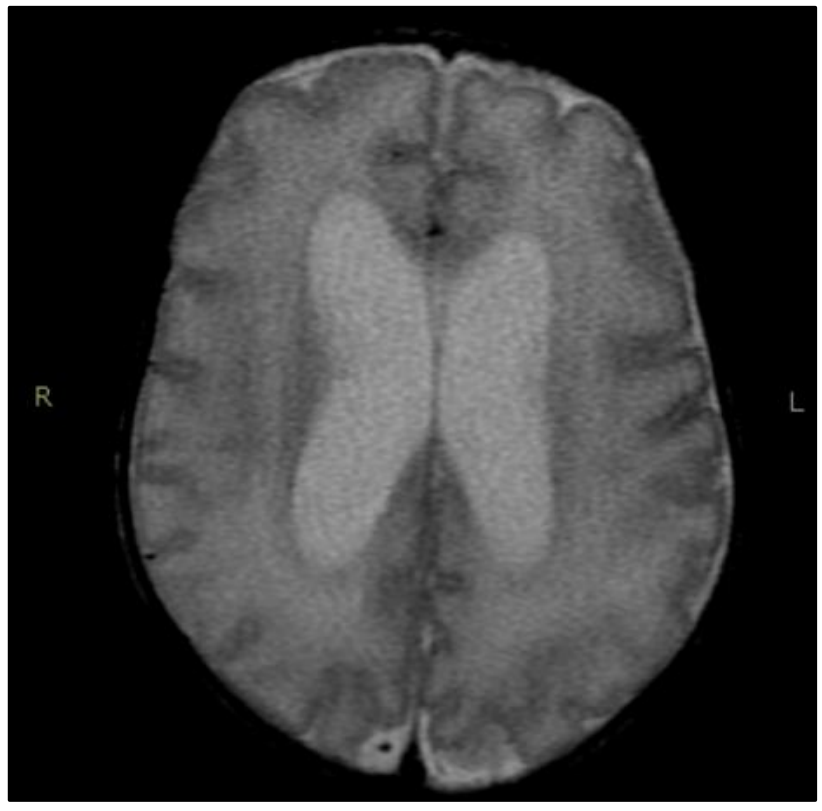

Fig. 9

\section{CASE 1}

Axial T2WI MR image showing hyperintense signal involving cortex and subcortical white matter of bilateral posterior water shed territories in a term baby.

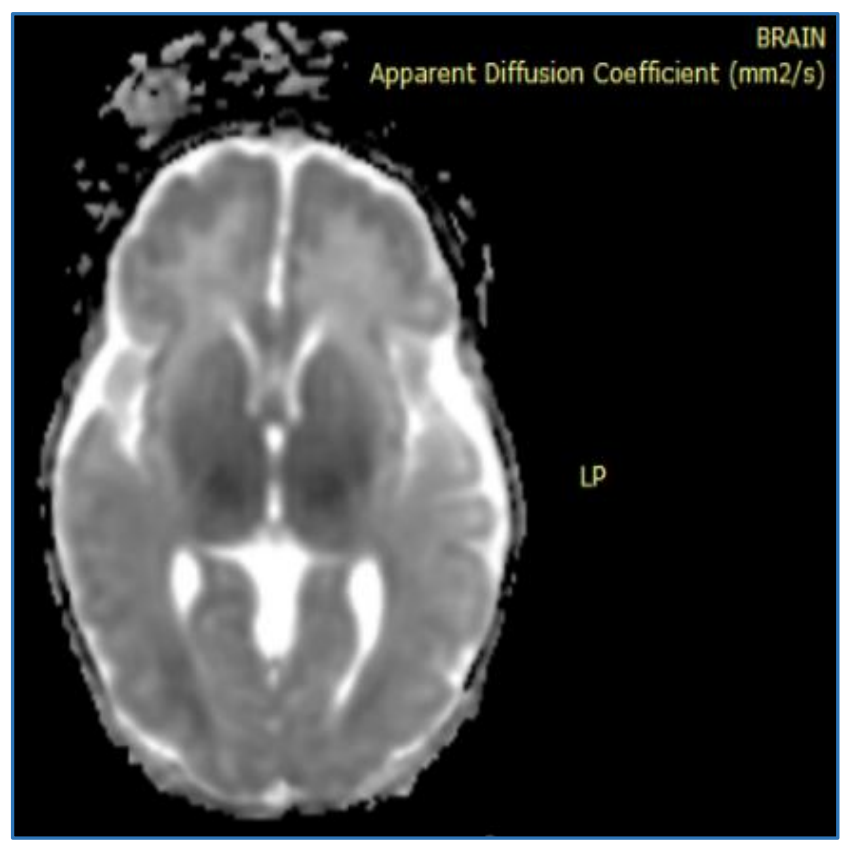

Fig. 10 
CASE 2

ADC image showing hypointense signal involving bilateral ventromedial thalami and left lentiform nucleus in a term baby with history of severe hypoxia.

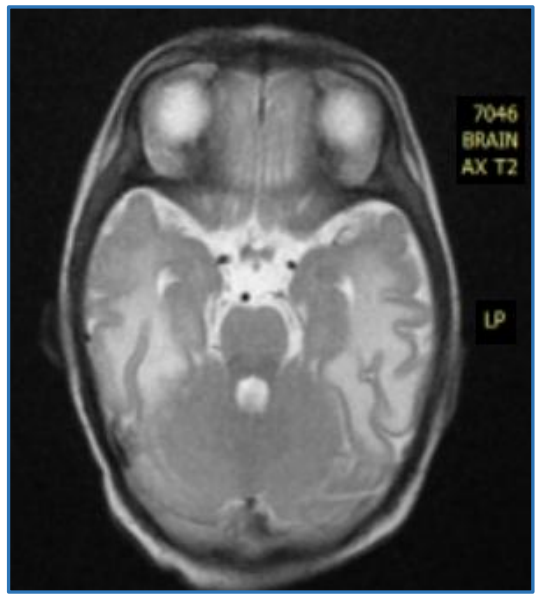

Fig. 11

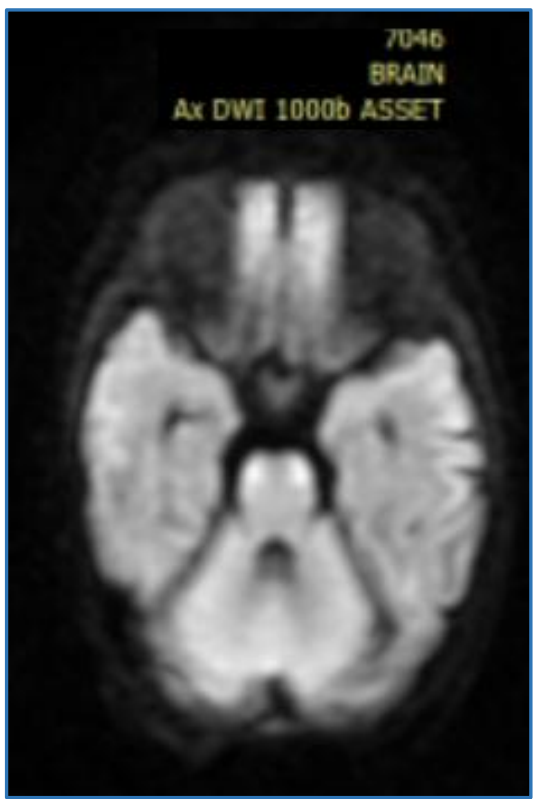

Fig. 12

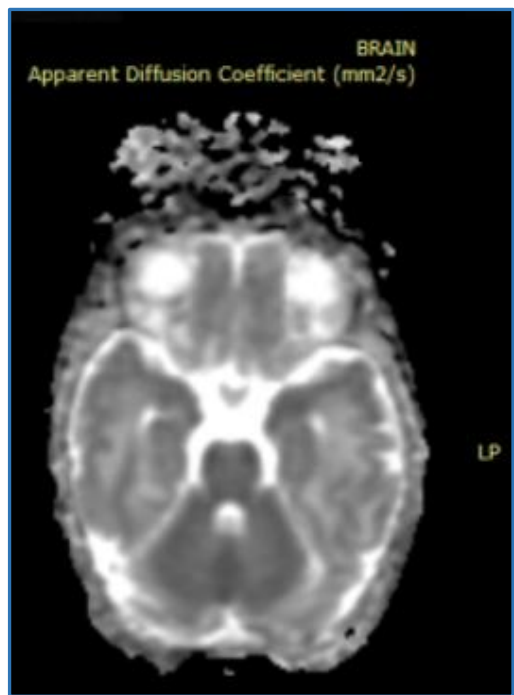

Fig. 13

\section{CASE 3}

Axial T2WI image (Fig. 3) in term neonate within 8 hrs. of delivery showing no abnormal signal. Diffusion weighted image (Fig. 4) showing hyperintensity in anterior pons in the same patient and ADC image (Fig. 5) showing hypointensity in the same region.

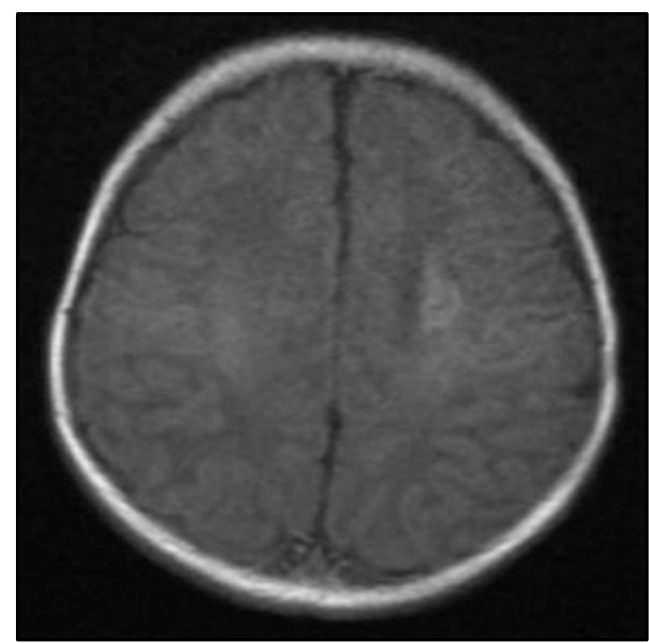

Fig. 14

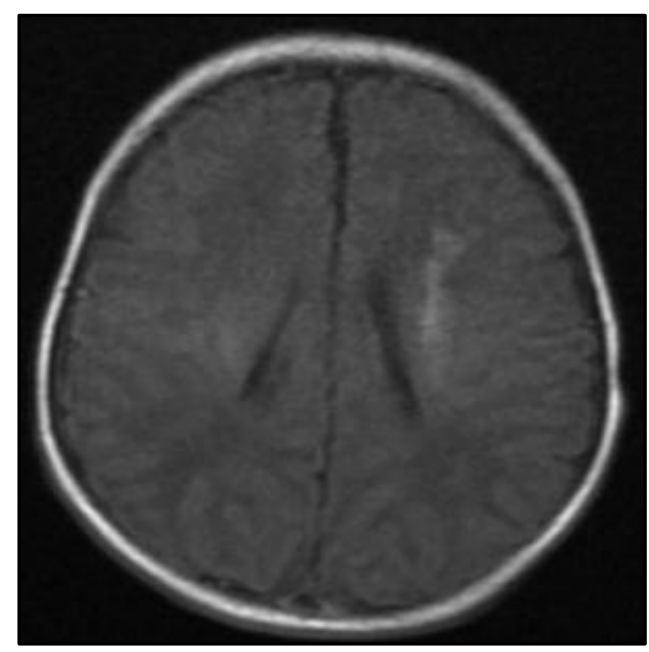

Fig. 15

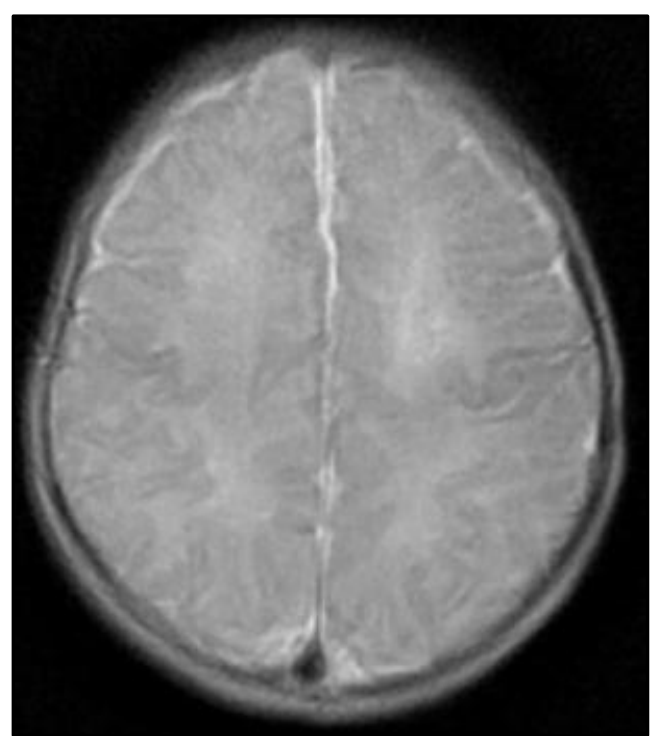

Fig. 16 


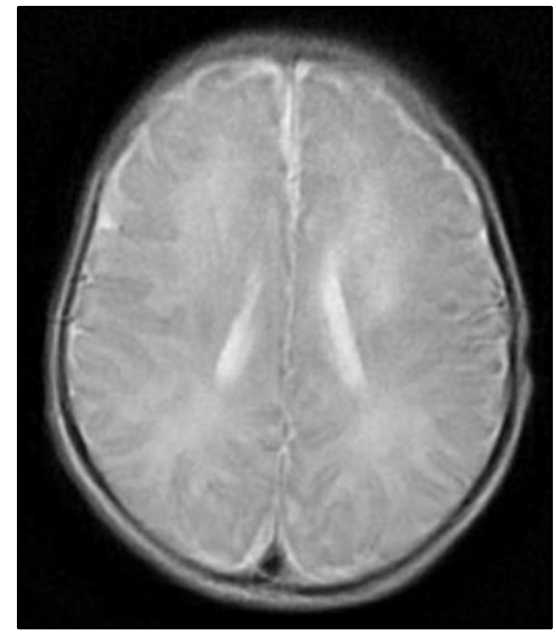

Fig. 17

CASE 4: Axial T1W images showing abnormal hyperintense signals in left perirolandic white matter axial T2W images of same patient showing abnormal hyperintense signal in the left perirolandic white matter.

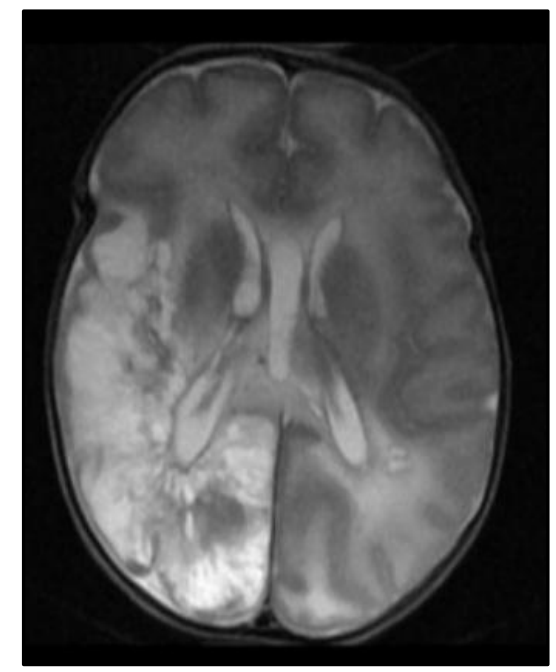

Fig. 18

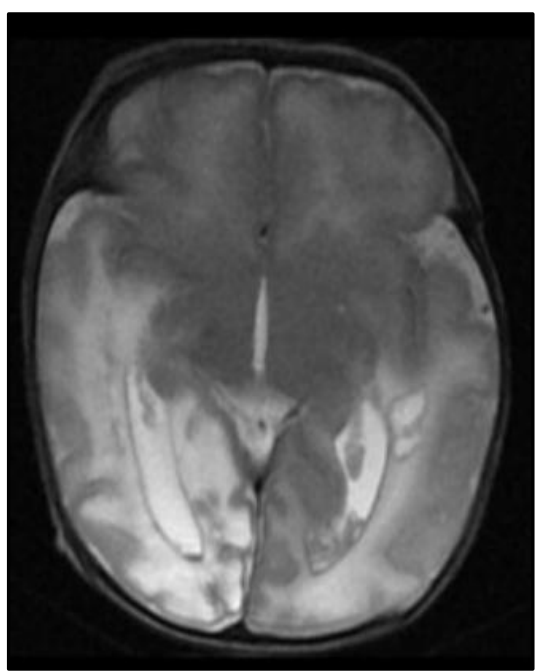

Fig. 19

\section{CASE 5}

Axial T2W images in a preterm neonate showing bilateral multicystic encephalomalacia $(\mathrm{R})>(\mathrm{L})$.

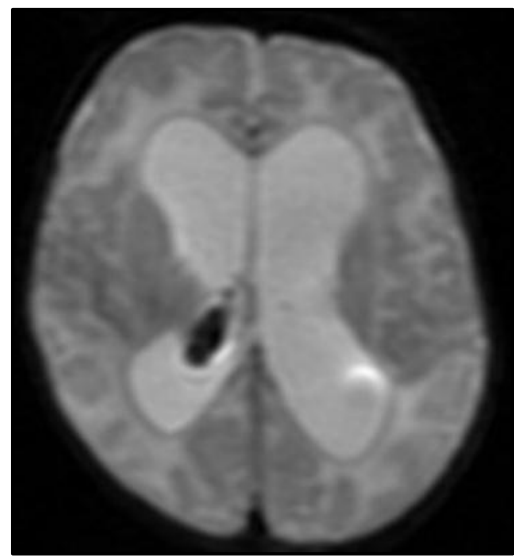

Fig. 20

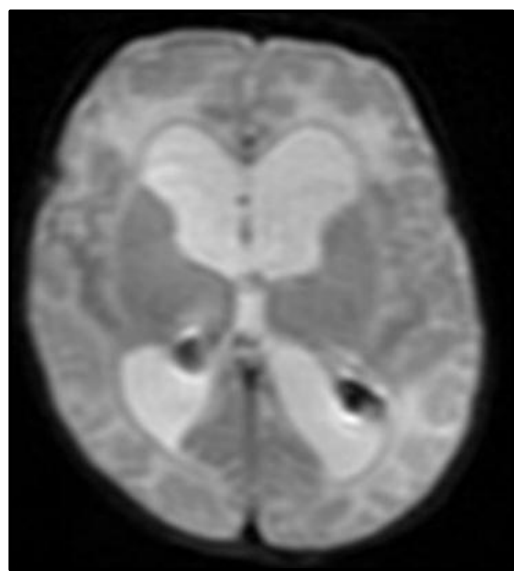

Fig. 21

\section{CASE 6}

Axial GRE images in a preterm neonate with germinal matrix bleed showing blooming artefacts.

\section{DISCUSSION}

After MR imaging, most of the Sarnat Stage I and II patients found in mild category and stage III patients found in severe category. Of the babies clinically considered having mild hypotension $>1 / 2(54.2 \%)$ of them were found to be normal after imaging. Of the babies clinically having moderate hypotension, $20 \%$ were normal at MRI; and $52 \%$ of them showed only mild changes and $12 \%$ showed severe involvement. Of those having profound hypotension more than half of them revealed primary involvement in thalamus, basal ganglia and brain stem. These observations were found in study conducted by Kaufman SA et al. ${ }^{2}$ Mild encephalopathy correlates with normal MRI result, severe encephalopathy correlates with abnormal basal ganglia and moderate encephalopathy manifested basal ganglia abnormality with a likelihood ratio of 0.785 .

\section{Severity of Involvement}

In term babies with mild HIE, $72.2 \%$ showed signal changes in SCW. This observation was similar to study by Van Den Bergh $\mathrm{R}$ et al and Takashima S, Tanaka K.3,4 They observed that term infants who suffer similar degrees of hypotension sustain injury in the watershed portions of the cerebral cortex and in the underlying subcortical and periventricular white matter. Classically, this change in injury pattern has been attributed to a changing location of the intervascular boundary zones 
("Watershed regions"). According to them, it will lead to significant cortical subcortical gliosis and atrophy of parasagittal watershed areas in term neonates.

We observed that a large proportion of term babies with mild hypotension (83.3\%) revealed changes in periventricular white matter. According to Rutherford et al,5 "It is sometimes difficult to differentiate periventricular changes due to immature white matter in infants under one year of age. The change may be due to delay in myelination, although in some infants they seem to become more obvious with time. Even though mild HIE is a clinical diagnosis and not an MRI diagnosis, as the changes are noted in infants who had already been diagnosed with HIE, such infants are to be monitored with follow-up imaging and detailed motor examination.

In the study by Sie $\mathrm{U}$ et al, 6 also found that multicystic encephalopathy in $70 \%$ preceded by mild hypoxic ischaemia. It is observed that those babies having profound asphyxia (Stage III), 50\% showed involvement of thalamus, 25\% showed involvement of basal ganglia and 25\% showed involvement of brainstem. Similarly, Volt et al ${ }^{7}$ found that thalamic-striatal damage is the hallmark of more widespread brain damage, which is more frequently observed in asphyxiated term babies. According to Barkovich et al, 8 primary brainstem, thalamus and basal ganglia involvement is revealed in those with cardiocirculatory arrest.

It is observed that those having moderate hypotension, $80 \%$ showed changes in PRWM and 60\% showed changes in PLIC. According to Rutherford MA and Pennock et al, ${ }^{9}$ abnormal MR signal in internal capsule predicts poor neurodevelopmental outcome in infants with HIE. This information can be helpful for evaluating the appropriateness of continuing life sustaining therapy and for advising parents.

It is found that in preterm babies with mild-moderate hypotension, periventricular white matter is the most common area affected and germinal matrix is most commonly affected in severe hypotension. It is also observed by Sie U et al, ${ }^{6}$ where PV leukomalacia in $82 \%$ after subacute or chronic hypoxia and in preterm babies.

\section{APGAR \\ In our study, it is found that of the babies with low Apgar at 5 minutes, $93 \%$ were affected and $50 \%$ of them showed severe involvement in MRI. And of the babies having normal Apgar, $64.2 \%$ were affected, majority (89\%) were mild and none of them showed severe involvement. In the study by Mercuri E,10 it is observed that cerebral infarction and scattered white matter changes were most common findings in infants with low Apgar 4 and above. Severe and moderate basal ganglia and thalamus lesions were observed in very low Apgar 3 or below. Even though Apgar scores did not always predict the motor outcome at 2 years of age, the preserved severity of the sequelae mainly reflected the site and severity of MR findings.}

\section{Seizure}

In our study, $60 \%$ cases had history of seizure and $84.4 \%$ showed involvement after MRI. We also observed that those with onset of seizure within 24 hrs. of birth, majority were mild and those with seizure after $24 \mathrm{hrs}$. majority were severe $(84.4 \%)$. We found out a statistically significant association between seizure and HIE with respect to time of onset. Studies conducted by Sankar JM11 and Zupane ML12 found that the most common cause of neonatal seizure is HIE.
In this study, percentage of abnormal MRI findings were more among low birth weight babies compared to normal birth weight babies and in male babies compared to female. However, it was not statistically significant.

\section{CONCLUSION}

- MR pattern of brain damage in patients with prolonged partial asphyxia is seen to evolve in a predictable manner corresponding to the known maturation of brain and its vascular supply.

- In this study, more than half of clinically mild cases and $20 \%$ of clinically moderate cases were found to be normal after MR imaging. Hence, this study signifies the value of MRI in excluding unaffected patients from those clinically considered as mildly or moderately asphyxiated and the need of MRI to be performed in all patients with clinical diagnosis of HIE by Sarnat's criteria.

- In this study, term babies with mild encephalopathy shows more involvement of periventricular white matter than subcortical white matter.

- Periventricular white matter involvement observed in term babies with clinically mild HIE may be due to immature zones of myelination or due to ischaemia itself. In such cases, follow-up imaging is advised later for confirmation.

- Clinically severe encephalopathy correlates with abnormal basal ganglia-thalamic lesions in term babies and germinal matrix haemorrhage in preterm babies.

- Clinically moderate encephalopathy correlates with signal changes in posterior limb of internal capsule and perirolandic white matter.

- This study shows statistically significant association between prematurity and severe brain involvement in HIE.

- $\quad$ This study highlights the importance of MRI in neonates with low Apgar at 5 mins., as these cases show severe involvement in MRI and there is statistically significant association between low Apgar score and brain injury in birth asphyxia.

- This study signifies the association between time of onset of seizure in asphyxiated neonates and severity of brain involvement.

- In this study, percentage of abnormal MRI findings were more among low birth weight babies compared to normal birth weight babies and in male babies compared to female. However, it was not statistically significant.

\section{REFERENCES}

1. Chao CP, Zaleski CG, Patton AC. Neonatal hypoxicischemic encephalopathy: multimodality imaging findings. Radio Graphics 2006;26:S159-S172 .

2. Kaufman SA, Miller SP, Ferriero DM, et al. Encephalopathy as a predictor of magnetic resonance imaging abnormalities in asphyxiated newborns. Pediatr Neurol 2003;28(5):342-6.

3. Van den BR. Centrifugal elements in the vascular pattern of the deep intracerebral blood supply. Angiologica 1969;20(2):88-98.

4. Takashima S, Tanaka K. Development of the cerebrovascular architecture and its relationship to periventricular leukomalacia. Arch Neurol 1978;35(1):11-16. 
5. Rutherford M, Pennock J, Zchwieso J, et al. Hypoxicischaemic encephalopathy: early and late magnetic image resonance findings in relation to outcome. Arch Dis Child 1996;75(3):F145-F151.

6. Sie LT, van der Knaap MS, Oosting J, et al. MR patterns of hypoxic-ischemic brain damage after prenatal, perinatal or postnatal asphyxia. Neuropediatrics 2000;31(3): 128-36.

7. Voit T, Lemburg P, Neuen E, etal. Damage of thalamus and basal ganglia in asphyxiated full-term neonates. Neuropediatrics 1987;18(3):176-81.

8. Barkovich AJ, Truwit CL. Brain damage from perinatal asphyxia: correlation of MR findings with gestational age. AJNR 1990;11(6):1087-1096.
9. Rutherford MA, Pennock JM, Counsell SJ, et al. Abnormal magnetic resonance signal in the internal capsule predicts poor neurodevelopmental outcome in infants with hypoxic-ischemic encephalopathy. Pediatrics 1998;102(2 Pt 1):323-8.

10. Mercuri E, Rutherford M, Barnett A, et al. MRI lesions and infants with neonatal encephalopathy. Is the Apgar score predictive? Neuropediatrics 2002;33(3):150-156.

11. Sankar JM, Agarwal R, Deorari A, et al. Management of neonatal seizures. Indian J Pediatr 2010;77(10):1129-35.

12. Zupanc ML. Neonatal seizures. Pediatr Clin North Am 2004;51(4):961-78, ix. 